\title{
Wave zone extraction of gravitational radiation in three-dimensional numerical relativity
}

\author{
David R. Fiske,, , 2, 田 John G. Baker, ${ }^{2}$ James R. van Meter, ${ }^{2}$ Dae-Il Choi, ${ }^{3,2}$ and Joan M. Centrella ${ }^{2}$ \\ ${ }^{1}$ Department of Physics, University of Maryland, College Park, Maryland 20742-4111 \\ ${ }^{2}$ Laboratory for Gravitational Astrophysics, NASA Goddard Space Flight Center, Greenbelt, Maryland 20771 \\ ${ }^{3}$ Universities Space Research Association, 10211 Wincopin Circle, Suite 620, Columbia, Maryland 21044
}

\begin{abstract}
We present convergent gravitational waveforms extracted from three-dimensional, numerical simulations in the wave zone and with causally disconnected boundaries. These waveforms last for multiple periods and are very accurate, showing a peak error to peak amplitude ratio of $2 \%$ or better. Our approach includes defining the Weyl scalar $\Psi_{4}$ in terms of a three-plus-one decomposition of the Einstein equations; applying, for the first time, a novel algorithm due to Misner for computing spherical harmonic components of our wave data; and using fixed mesh refinement to focus resolution on non-linear sources while simultaneously resolving the wave zone and maintaining a causally disconnected computational boundary. We apply our techniques to a (linear) Teukolsky wave, and then to an equal mass, head-on collision of two black holes. We argue both for the quality of our results and for the value of these problems as standard test cases for wave extraction techniques.
\end{abstract}

PACS numbers: 04.25.Dm, 04.30.Nk, 04.30.Db

\section{INTRODUCTION}

At the present time, several ground-based gravitational wave detectors, using laser interferometry, are already or very near to operating [1, 2, 3], and the NASA-ESA space-based antenna LISA is scheduled to be launched in the next decade [4]. These experiments should provide the first direct probe of strong-field gravitational physics. The data analysis needs of these observations, however, require accurate waveform templates for use in matched-filtering algorithms. While the early and late stages of a merger process can be treated analytically using post-Newtonian and perturbation theory, respectively, the highly dynamical merger period can only be understood with the full, non-linear Einstein equations. In this latter regime, numerical relativity is essential [3].

Computing waveforms from three-dimensional numerical simulations has, however, proven challenging [5, 6, 7, 8]. One of the primary problems is that there are a variety of length scales in a typical problem with sources. For a binary black hole collision, for example, the dynamics of the merger scale with the masses and spins of the black holes, while the waves generated by those dynamics have length scales that are one or two orders of magnitude larger. In addition, the boundary conditions applied at the edges of the computational domain tend to generate spurious ingoing radiation (which may or may not satisfy the full Einstein equations), which can easily contaminate wave signals unless the boundary is causally disconnected from the place and time at which the signal is desired. In addition, one must choose a stable form of the $3+1$ Einstein equations, supplemented by an appropriate set of gauge conditions, in order to build a code

\footnotetext{
${ }^{*}$ Current Address: Decisive Analytics Corporation, 1235 South Clark Street, Arlington, VA 22202
}

(cf. Ref. [9]). Only recently has it become possible to mix all of these components into a single simulation in three dimensions. (See Refs. 10, 11] for very recent examples.)

In this paper, we bring together a variety of techniques and technologies to successfully simulate dynamical spacetimes; to compute, via the Weyl scalar $\Psi_{4}$, a gauge invariant measure of gravitational radiation; to analyze that radiation quantity through spherical harmonic decomposition; and to demonstrate that our approach not only converges very well, but that it also is very accurate. For our sample linear problem, a Teukolsky wave propagating on a flat background, we see peak error to peak amplitude ratios on the order of $0.4 \%$, and for our sample non-linear problem, the head-on collision of two equal mass black holes, the same ratio is on the order of $2 \%$ or less. These waveforms maintain this level of accuracy for several periods.

While we present our results, we also wish to lay out some well defined examples for future use as testbeds for wave extraction schemes, much in the spirit of the "Apples with Apples" tests for numerical relativity [12]. ${ }^{1}$ To this end, we attempt to abstract away, where possible, from the details of our Einstein solver, and focus on (1) the higher level details of how we define and compute gravitational radiation quantities given numerically evolved solutions to the Einstein equations, (2) the description of a logical progression of test cases for validating results, and (3) the enumeration of analytic solutions, symmetries, and cross-checks between test cases that lead, when taken together, to a high level of confi-

\footnotetext{
${ }^{1}$ While this paper was in final preparation, Ref. [1] was released as a preprint, and, indeed, used very similiar test cases for wave extraction. Ref. 11] focuses primarily, but not exclusively, on extractions via the Zerilli-Moncrief formalism rather than the Newman-Penrose formalism that we use here.
} 
dence in both the methods used and the results obtained.

To these ends, we carefully describe our particular approach to the problems of computing and analyzing gravitational waveforms in Section [II In that section, we describe our method for computing the Weyl scalar $\Psi_{4}$ from our $3+1$ numerical data, describe our particular algorithm for computing spherical harmonic components of the Weyl scalar, and briefly describe our Einstein solver. In Section IIII we bring this machinery to bear on the Teukolsky wave spacetime. This is a linear problem with an analytic solution, which we use to validate our code and our methods. We then turn to a non-linear problem, the head-on collision of a binary black hole system in Section [V] which more closely resembles astrophysically interesting sources and tests our methods on a non-linear problem. We conclude with some discussion of our results in Section $\nabla$ The appendices contain more details about the spherical harmonic decomposition algorithm and about the Teukolsky spacetime.

\section{METHODOLOGY}

Computing gravitational waves from numerical simulations of the Einstein equations requires combining a variety of nearly independent mathematical formalisms and numerical techniques into a single code. The methods that we present, implement, and test here are not specific to any particular formalism or to our code. They are made explicit in Section IIA where we define gravitational radiation via the Weyl scalars in the NewmanPenrose formalism, and in Section [IIB where we discuss the need and a method for computing spherical harmonic components of radiation data. In Section IIC we briefly discuss our particular Einstein solver, with which we carry out numerical simulations to validate our method.

\section{A. Weyl Scalars}

We use the Newman-Penrose formalism to compute gravitational radiation quantities. In this formalism, one chooses a tetrad of four null basis vectors, which are conventionally labeled $l^{a}, n^{a}, m^{a}$, and $\bar{m}^{a}$. (Note that, although our code uses a $3+1$ decomposition of the Einstein equations, indices on Newman-Penrose quantities are always understood to run over four dimensional spacetime indices $a=0,1,2,3$.) Of these vectors, $l^{a}$ and $n^{a}$ are real and point along "outgoing" and "ingoing" directions, respectively. The vectors $m^{a}$ and $\bar{m}^{a}$ span "angular" directions and are complex conjugates of each other. These vectors are always chosen to satisfy the orthogonality conditions

$$
l^{a} m_{a}=n^{a} m_{a}=0 .
$$

In addition, we impose the normalization conditions $l^{a} n_{a}=-1$ and $m^{a} \bar{m}_{a}=+1$, which are used by most, but not all, authors (cf. Ref. [13]).

Given a tetrad, tensor quantities can be recast as sets of coordinate scalars by projecting tensor components onto the basis. Applying this procedure to the Weyl tensor $^{2} C_{p q r s}$ yields five complex scalars. This is particularly useful for gravitational wave studies since one of the five,

$$
\Psi_{4}=-C_{p q r s} n^{p} \bar{m}^{q} n^{r} \bar{m}^{s},
$$

represents outgoing gravitational waves. Indeed, the primary goals of this paper are to construct $\Psi_{4}$ in some sample, numerically evolved spacetimes, and to demonstrate that such computations are accurate characterizations of the wave content of the spacetime.

Since, in our code, we have only $3+1$ quantities on a single time slice from which to compute the four dimensional Riemann tensor, we follow Ref. [14] and write

$$
\begin{aligned}
& { }^{(4)} R_{i j k l}=R_{i j k l}+2 K_{i[k} K_{l] j} \\
& { }^{(4)} R_{0 j k l}=-2\left(K_{j[k, l]}+\Gamma_{j[k}^{m} K_{l] m}\right) \\
& { }^{(4)} R_{0 j 0 l}=R_{j l}-K_{j m} K_{l}^{m}+K K_{j l}
\end{aligned}
$$

which express four dimensional quantities on the left hand sides in terms of $3+1$ quantities on the right. ${ }^{3}$ Here the indices are spatial $(i, j, k, l, m=1,2,3), K_{i j}$ is the extrinsic curvature tensor for a spatial slice as embedded in the full spacetime manifold, and $K$ is its trace. The symbols $\Gamma_{j k}^{m}, R_{i j k l}$, and $R_{j l}$ stand for, respectively, the connection coefficients, Riemann tensor, and Ricci tensor associated with the three-dimensional spatial metric on the slice.

\section{B. Spherical harmonic decomposition}

In our simulations, we would like to be able to extract the spherical harmonic components of gravitational waves. We find this very valuable when analyzing the data because

1. Numerical noise tends to have higher angular frequency than genuine wave signals, and is therefore filtered by the decomposition process.

2. A priori knowledge about symmetries in the data or dominant modes associated with physical processes allow important checks on the plausibility of numerical solutions (especially when exact solutions are not available).

\footnotetext{
${ }^{2}$ In vacuum, which is the only case that we consider here, the Weyl tensor is equal to the Riemann tensor.

3 There are two errors in Ref. [14] associated with what we call Eq. (3). First there is a factor of two difference between Eq. (3b), which is correct, and the corresponding equation in Ref. 14]. Second, Eq. (3) in this paper properly reflects the fact that the left hand sides refer to the four dimensional Riemann tensor.
} 
3. Some characteristics of gravitational radiation, such as quasi-normal modes for instance, are best understood in terms of spherical harmonic components.

In practice, however, we face a technical problem in computing spherical harmonic components of our data. While our data is stored on a cubic grid, the spherical harmonic components

$$
{ }_{s} \Phi_{l m}(t, r)=\oint_{s} \bar{Y}_{l m}(\theta, \phi) \Phi(t, r, \theta, \phi) d \Omega
$$

of a general (complex valued) function $\Phi$ in a spherical harmonic basis with spin-weight $s$ are defined by an integral over a sphere of radius $r$. Computing the integral in Eq. (4) requires some type of interpolation from grid points to points on that sphere.

One could solve this problem by the straightforward method of first interpolating the grid function $\Phi$ to points on the sphere, and then using some numerical approximation to the integral in Eq. (4). This process of interpolation followed by integration would then need to be performed at each time, at each radius, and for every function for which the spherical harmonic components are desired.

We adopt a different algorithm due to Misner. Following Ref. 15], we smear the surface integral in Eq. (44) into a volume integral over a shell of half-thickness $\Delta$, and create an orthonormal basis for functions on this shell by combining the spherical harmonics in the angular directions with the first $N$ Legendre polynomials $P_{n}$ in the radial direction. This approach, which we describe in more detail in Appendix $\mathrm{A}$ has the advantage that, given the parameters $\Delta$ and $N$, one need only compute a relatively small number of weights to carry out the volume integral, and that these weights depend only on the grid structure and the radius of extraction. This means that the weights can be computed once at the beginning of a simulation and stored. Further interpolations are not needed, and the weights are valid for all functions. (The weights do depend on the grid structure, so they would needed to be recomputed after grid structure changes in a simulation using adaptive mesh refinement.)

The question of how to choose the input parameters $\Delta$ and $N$ was addressed in Refs. [16, 17]. Under the assumption (motivated by the analysis in the references) that $\Delta$ is chosen proportional to the grid spacing, the parameter $N$ controls the order of convergence of the decomposition algorithm, while the parameter $\Delta$ controls the size of the error at that order. This analysis leads to the

Rule of Thumb: Choose $N$ just large enough to ensure that the error term proportional to $\Delta$ is not of leading order in the grid spacing $h$. Choose $\Delta$ just large enough to safely resolve $P_{N}$ on the shell.

For second order accurate codes such as ours, Refs. 16, 17] suggest choosing $N=2$ and $\Delta=3 h / 4$ based on this rule and empirical experiments. In cases where the shell passes through multiple refinement regions, the grid spacing of the coarsest grid through which the shell passes should be chosen to ensure that $P_{N}$ is resolved on both sides of the interface. We follow these suggestions for all work presented here.

\section{The Hahndol Code}

Our code is a fully three-dimensional, non-linear Einstein solver. We use a fairly standard formulation of the $3+1$ Einstein evolution equations known as BSSN [18, 19]; our particular implementation was described in detail in Ref. [20]. Because the formulation of the equations is not of primary interest here, and because the basics of the BSSN system are widely known, we do not focus on these equations.

The code uses second-order accurate finite differencing to approximate spatial derivatives and the iterative Crank-Nicholson method 21] to integrate the evolution equations forward in time. In both our previous and current work, we have verified second order convergence in our results.

Our parallel code uses the PARAMESH libraries 22] to handle domain decomposition and inter-processor synchronization. In addition, these libraries enable us to use non-uniform grids to focus computational resources in specific areas of the computational domain. Although the libraries support, and we look forward to using, the ability to adaptively modify the grid structure during the course of a simulation (adaptive mesh refinement), we currently fix our grid structures in advance using a priori estimates of where to focus resolution (fixed mesh refinement). Our code shows $92 \%$ of optimal scaling, up to 256 processors, when simultaneously doubling the number of processors used for a simulation and keeping the number of data points per processor constant [20].

Previous studies showed that the Hahndol code is able to propagate linearized gravitational waves (defined in terms of metric components using Teukolsky's solution [23]) across mesh refinement boundaries [24], and that the same code can handle strong, dynamical potentials moving across refinement regions [20]. In this paper we again focus on wave propagation, this time using a more formal definition of gravitational radiation, the Weyl scalars in the Newman-Penrose formalism, and on analyzing such data in a meaningful way.

\section{TEUKOLSKY WAVES}

Our first problem studies Teukolsky's solution 23] to the linearized Einstein equations, which represents a weak gravitational wave propagating through space. The linear nature of the initial data makes this an excellent first test problem for two reasons. First, the there is a 
analytic solution for all times, allowing a direct calculation of numerical errors for convergence and accuracy studies. Second, because this solution consists of a linear wave in the initial data, we are able to extract our waveforms at small radii and short evolution times. This second fact is extremely useful for debugging algorithms, and also allows higher resolutions for a fixed problem size since the volume of the computational domain need not be as large as it would in problems (like the head-on collision described in Section [V] in which the waves are generated by non-linear sources and must propagate to the wave zone before being extracted.

\section{A. Analytic Preliminaries}

Although the Teukolsky solution is well-known, we summarize it here for completeness and to establish notation. The general form of the spacetime metric

$$
\begin{aligned}
d s^{2}= & -d t^{2}+\left(1+A f_{r r}\right) d r^{2} \\
& +2 B f_{r \theta} r d r d \theta+2 B f_{r \phi} r \sin \theta d r d \phi \\
& +\left(1+C f_{\theta \theta}^{(1)}+A f_{\theta \theta}^{(2)}\right) r^{2} d \theta^{2} \\
& +2(A-2 C) f_{\theta \phi} r^{2} \sin \theta d \theta d \phi \\
& +\left(1+C f_{\phi \phi}^{(1)}+A f_{\phi \phi}^{(2)}\right) r^{2} \sin ^{2} \theta d \phi^{2}
\end{aligned}
$$

is given in terms of angular functions $f_{i j}$ and functions $A, B, C$. The angular functions for the $l=2, m=0$ (spin-weight -2 ) case that we consider here are given by Eqs. (B1) in Appendix B] The remaining functions, given by Eqs. (B2), are written in terms of a free generating function $F$.

We follow Choi et al. 24] in choosing

$$
F(x)=\frac{\mathcal{A} x}{\lambda^{2}} e^{-x^{2} / \lambda^{2}}
$$

as the exact form of the generating function, where the free parameters $\mathcal{A}$ and $\lambda$ represent the amplitude and the width of the wave respectively. The natural length unit in the problem is $\lambda$, and we consistently choose $\mathcal{A}=$ $2 \times 10^{-6} \lambda$. Moreover we take an equal superposition of an ingoing wave and an outgoing wave, both centered at the origin, for the initial data. This particular choice has a moment of time symmetry that allows us to set the extrinsic curvature tensor to zero in the initial data.

Choosing $F$ of the form in Eq. (6) gives a waveform with oscillations but of essentially compact support. This is ideal for testing codes with boundaries (both internal and external) because it makes clear when the wave passes through those boundaries, and allows one to easily detect any reflections that occur due to poor interface conditions; cf. Ref. [24].

For our $3+1$ code, we use this analytic solution to generate the initial data, and evolve forward in time using the gauge conditions lapse $\alpha=1$ and shift $\beta^{i}=0$.
We use the Kinnersley tetrad [25]

$$
\begin{aligned}
l & =\frac{1}{\Delta}\left(r^{2}+a^{2}, \Delta, 0, a\right) \\
n & =\frac{1}{2 \Sigma}\left(r^{2}+a^{2},-\Delta, 0, a\right) \\
m & =\frac{1}{\sqrt{2}(r+i a \cos \theta)}(i a \sin \theta, 0,1, i \csc \theta)
\end{aligned}
$$

to extract the waves in this linear wave problem. ${ }^{4}$ Eqs. (7) give the tetrad in terms of the Boyer-Lindquist coordinates for a Kerr black hole. In these equations, $\Delta=r^{2}-2 M r+a^{2}, \Sigma=r^{2}+a^{2} \cos ^{2} \theta, M$ is the mass of the Kerr black hole, and $a$ is its spin. Because BoyerLindquist coordinates are compatible with the coordinate system used for the Teukolsky metric (Eq. (5)) when $M=a=0$, we find this coordinate expression for the tetrad completely acceptable for this problem.

For this relatively simple spacetime, one can compute analytically the value of the Newman-Penrose quantity

$$
\Psi_{4}=\frac{\sin ^{2} \theta}{16}\left[-12 \frac{\partial^{2} C}{\partial t^{2}}+6 \frac{\partial^{2} A}{\partial t^{2}}+r\left(3 \frac{\partial^{3} B}{\partial t^{3}}+\frac{\partial^{3} A}{\partial t^{3}}\right)\right]
$$

which represents outgoing gravitational radiation. Noting that

$$
\begin{aligned}
{ }_{-2} Y_{20}(\theta, \phi) & =\sqrt{\frac{15}{32 \pi}} \sin ^{2} \theta \\
& =\sqrt{\frac{5}{6}}\left(Y_{00}(\theta, \phi)-\frac{1}{\sqrt{5}} Y_{20}(\theta, \phi)\right)
\end{aligned}
$$

it is clear that, as claimed, Eq. (8) is a pure $l=2, m=0$ mode.

Note also that Eq. (9b) provides a way to compute a spin-weight -2 spherical harmonic component from the usual (spin-weight 0) spherical harmonic components. We make use of this in the results presented here because our current implementation of the Misner algorithm computes only spin-weight 0 components, which suffices for our current work. In the near future we will have the capability to compute spin-weight -2 components directly.

\section{B. Numerical Results}

We evolved initial data specified by the Teukolsky solution described in Section $\amalg$ III on a domain with outer boundaries at $32 \lambda$ using a mesh with fixed refinement levels having a "box-in-box" structure centered on the origin. The boundaries were at $2 \lambda, 4 \lambda, 8 \lambda$, and $16 \lambda$; neighboring regions differed in resolution by a factor of

\footnotetext{
${ }^{4}$ The first component of $m^{a}$ should have the $\sin \theta$ term in the numerator as in Eq. 77); the corresponding equation in Ref. 14] is incorrect.
} 
two, with the finest regions surrounded by coarser regions. We need to apply a matching condition at the interfaces between grids of different resolutions; we use the algorithm described in Ref. [20]. We exploit the symmetry of the solution to reduce our computational burden by evolving only one octant of our data and using appropriate symmetry boundary conditions to mimic a full grid. In order to compute convergence factors, we ran the code at innermost resolutions $\lambda / 24$ and $\lambda / 48$. The wave propagates outward from the origin, crossing the refinement boundaries in turn. We then attempted to compute the spherical harmonic components of that wave at five distinct spherical "detectors" located at $r=3 \lambda, 4 \lambda, 5 \lambda$, $6 \lambda$, and $7 \lambda$.

To help visualize how the refinement boundaries and the extraction radii are related geometrically, we find it useful to draw two dimensional extraction maps, as shown in Fig. 1 The extraction maps make it clear that the shells used to compute the spherical harmonic components generically pass through multiple refinement regions, especially since in three dimensions the corners of the cubic refinement regions tend to pass through the spherical extraction shells. The extraction maps shown in Fig. 1]correspond to the innermost $(r=3 \lambda)$ extraction radius and the $r=5 \lambda$ extraction radius in two sample resolutions. (Extraction maps for nearly all extraction radii and two resolutions appear in Ref. 16].) Note that only the innermost refinement regions are shown in the maps. In each case, the entire map was surrounded by additional (coarser) refinement regions.

We present our numerical waveforms in Panel A of Fig. 2] which shows the $l=2, m=0$ component of $\Psi_{4}$ for a single wave as computed at our five distinct extraction radii. The amplitude scales approximately as $1 / r$, which is the correct behavior to leading order in $1 / r$, and the shape of the wave remains consistent as it moves outward. The consistency between the waveforms as extracted at the various radii is demonstrated convincingly in Panel B, in which we have scaled-up the waveforms by a factor or $r$ and shifted them in time to $t=3 \lambda$. Assuming a unit speed of propagation, this should, up to sub-leading terms in $1 / r$, result in overlaying waveforms. Indeed the agreement demonstrated in Panel B is striking.

In evaluating the effects of the refinement boundaries on the spherical harmonic decomposition algorithm, one should bear in mind that no two of the extraction radii have the same geometric relationship with the refinement boundaries (cf. Fig. (1), and yet they nonetheless generate perfectly consistent results.

Since we know the analytic solution for this test problem, we were able to make a detailed convergence study with two resolution levels. We verified that not only the waveforms themselves but also the extracted spherical harmonic components converge at second order, even with the extraction spheres passing through the refinement boundaries, and that the solution is highly accurate. Fig. 3 shows the errors, scaled by appropriate pow-
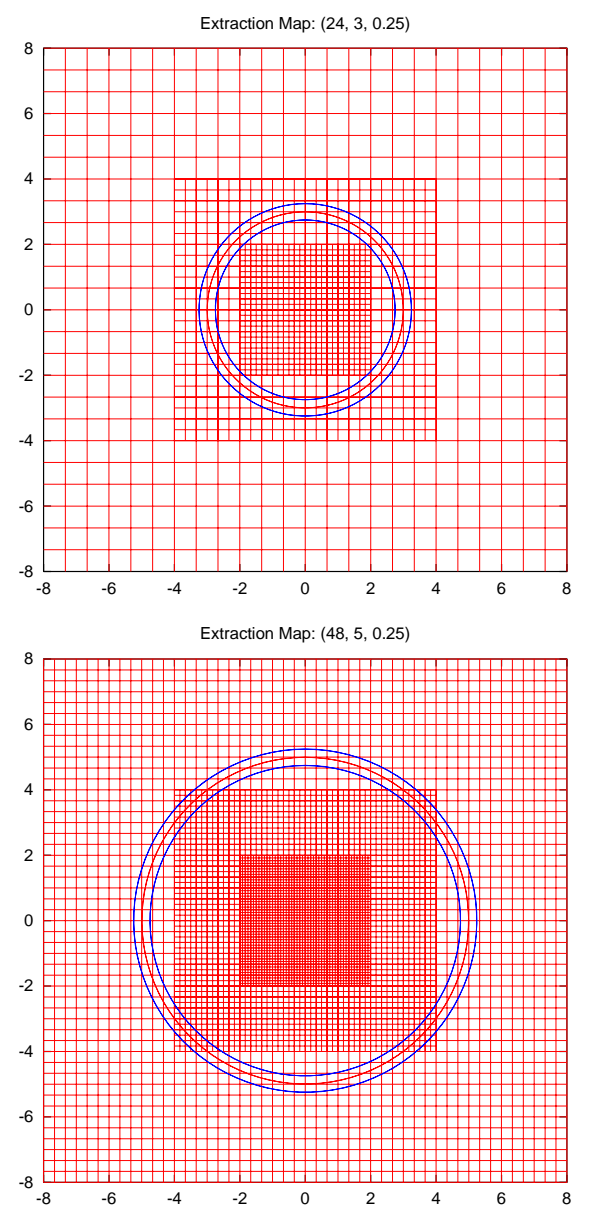

FIG. 1: Selected extraction maps. Each map shows the computational grid in a coordinate plane and is labeled with a triple of numbers $(N, R, \Delta)$, which indicate the number of (cell-centered) grid points across one coordinate direction in the coarsest region, the extraction radius, and the halfthickness of the shell, respectively. Lengths are measured in units of $\lambda$. Note that the shells generically pass through multiple refinement regions, especially since, in three dimensions, the corners of the cubic refinement regions tend to poke through the spherical extraction shells. The three circles drawn on each graph show the extraction sphere (red) and the edges of the finite-thickness shell (blue) around the sphere used in the Misner algorithm.

ers of two to indicate second-order convergence, of the numerical results as compared to the analytic solution. It is clear from the figure that we do have second order convergence. Note from Fig. [3 that our peak error in our highest resolution simulation is approximately $6 \times 10^{-7}$ for a waveform with peak amplitude of approximately $1.5 \times 10^{-4}$ (cf. Panel A of Fig. 2), giving a fractional error of approximately $0.4 \%$. 


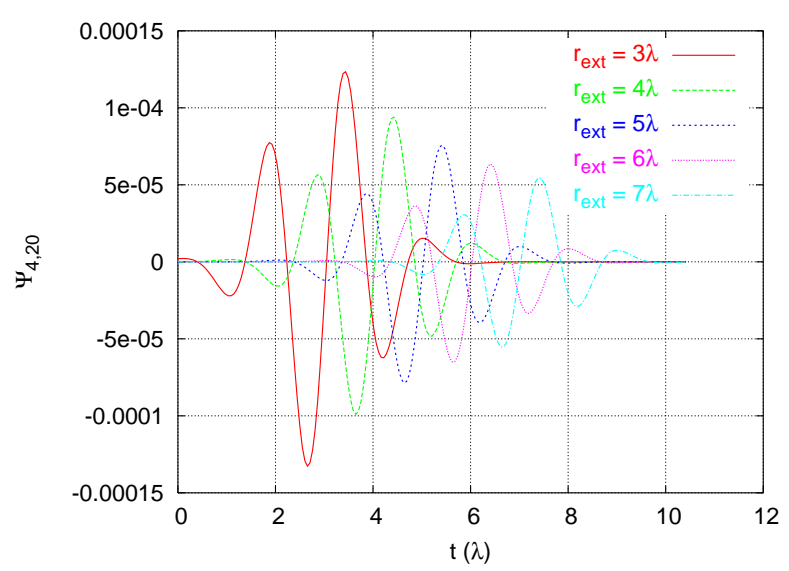

(A)

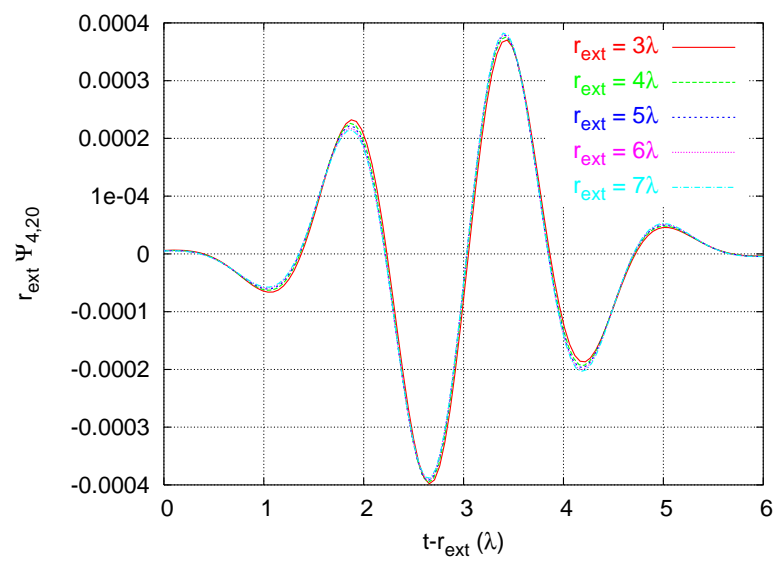

(B)

FIG. 2: The $l=2, m=0$ (spin-weight -2 ) component of the Teukolsky wave, as computed at five different radii for the highest resolution run. The waveform is preserved up to the leading order $1 / r$ scaling. Panel $\mathrm{A}$ shows the raw data. Panel B shows the same data, scaled-up by $r_{\text {ext }}$ and shifted in time to $t=3 \lambda$, the location of the inner-most extraction radius.

\section{BLACK HOLE HEAD-ON COLLISION}

We also tested our techniques on a well-studied nonlinear problem, the equal-mass, head-on collision of two black holes [10, 11, 26, 27]. This problem is more closely related to the astrophysical problems of interest to us and, consistent with our goal of testing our techniques, allows a variety of symmetries that we can use as checks on our numerical solutions.

\section{A. Analytic Preliminaries}

In our head-on collisions, we place two black holes, each of mass $M / 2$, on the $z$ axis at a coordinate distance of $1.1515 \mathrm{M}$ from the origin. The initial data is generated by the puncture prescription of Ref. [28], which is a generalization of Brill-Lindquist initial data [29]. We then

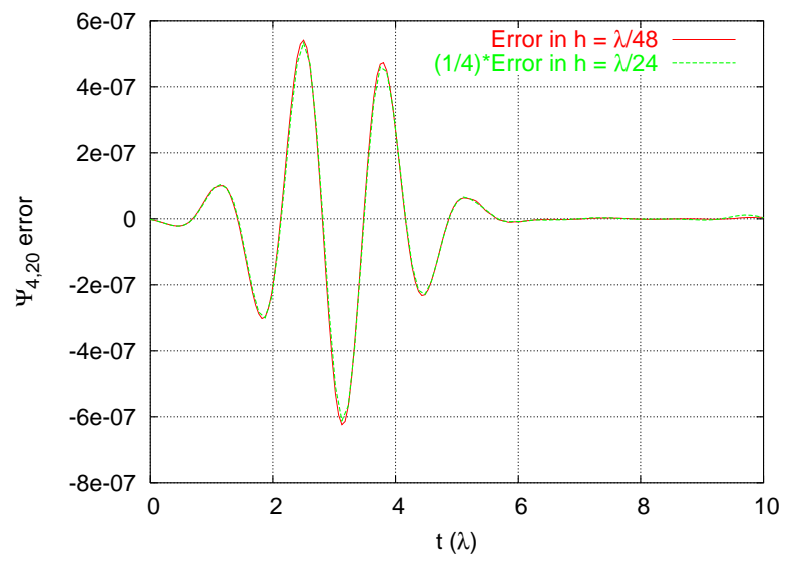

FIG. 3: A convergence plot for the Teukolsky wave simulation. The lines are the errors in the $l=2, m=0$ (spin-weight -2) component of the simulations at the two resolutions as compared to the analytic solution.

use the excision algorithm of Ref. [30] to avoid evolving the portions of our grid interior to the (initially separate) apparent horizons of our initial data. We neither allow our excision regions to move nor switch to a single, larger excision region after a common horizon forms. Although one or both of these techniques will likely be desirable in future simulations, we find the simple approach sufficient to extract convergent waveforms in this case.

Once we generate our initial data, we evolve it in our $3+1$ code using the $1+\log$ evolution equation

$$
\frac{\partial \alpha}{\partial t}=-2 \alpha K
$$

for the lapse $\alpha$, and the hyperbolic Gamma-driver evolution equations

$$
\begin{aligned}
\frac{\partial \beta^{i}}{\partial t} & =\frac{3}{4} \alpha \psi_{B L}^{-4} B^{i} \\
\frac{\partial B^{i}}{\partial t} & =\frac{\partial \tilde{\Gamma}^{i}}{\partial t}-\eta B^{i}
\end{aligned}
$$

for the shift $\beta^{i}$. In these equations, $K$ is the trace of the extrinsic curvature tensor for our slice, $\tilde{\Gamma}^{i}$ is the conformal connection function of the BSSN evolution system (cf. Ref. 19]), and $B^{i}$ is defined by Eq. (11a) to make the gauge evolution equations first order in time. The quantity

$$
\psi_{B L}(r)=1+\sum_{i=1}^{N} \frac{M_{i}}{2\left|\vec{r}-\vec{r}_{i}\right|}
$$

is the Brill-Lindquist factor used to generate puncture initial data for $N$ black holes with masses $M_{i}$ and positions $\vec{r}_{i}$. (In our case, $N=2$ and $M_{1}=M_{2}=M / 2$.) In our work, we choose the parameter $\eta=2.8 / M$, and set $\alpha=1$ and $\beta^{i}=0$ at the initial time. These gauge conditions were first studied in Ref. [9]. 
For the head-on collision we do not use the Kinnersley tetrad, since we have no way of knowing that the coordinate expressions in Eq. (7) are appropriate to this numerically evolved spacetime. (Indeed, for a general problem, one would assume that they are not appropriate.) Instead, we construct an orthonormal tetrad from the numerically evolved spacetime using a Gram-Schmidt procedure as described in Ref. [14]. ${ }^{5}$

Since we do not have an analytic solution for the Weyl scalars for the head-on case, we cannot directly compute the error in our solution. The symmetries of the problem nonetheless provide us with several analytic checks on our numerical results. Before discussing these symmetries specifically, however, it is worth making explicit a related point: There are three independent symmetry axes in this (and any axisymmetic) problem. The first is the symmetry axis of the physical problem, i.e. the axis along which the two black holes collide. The second is the axis with respect to which the tetrad is computed. (Careful examination of the Kinnersley tetrad defined by Eq. (7), for example, reveals this explicitly in coordinate expressions.) The third is the axis with respect to which we compute the spherical harmonics. Only if we align all of these axes of symmetry (conventionally along the $z$ axis) will all of our symmetry checks be true. ${ }^{6}$

Assuming that, as in the problem described here, all three symmetry axes are aligned along the $z$-axis, the numerical solution should have the following properties:

- $\operatorname{Re}\left\{\Psi_{4}\right\}$ is axially symmetric and is symmetric under the transformation $z \rightarrow-z$ up to the round-off level.

- $\operatorname{Im}\left\{\Psi_{4}\right\}$ converges to zero.

- Viewed in a spin-weight -2 basis, the dominant contribution to $\operatorname{Re}\left\{\Psi_{4}\right\}$ comes from the $l=2, m=$ 0 mode.

- Viewed in a spin-weight 0 basis, truncation level errors in $\operatorname{Im}\left\{\Psi_{4}\right\}$ appear in only in modes with odd values of $l \geq 3$ and even values of $m \neq 0$.

\footnotetext{
${ }^{5}$ We used the Teukolsky wave as a check on our implementation of the Gram-Schmidt construction. In the limit of perturbed flatspace, the Gram-Schmidt procedure recovers the Kinnersley tetrad up to a trivial rescaling of $l^{a}$ and $n^{a}$. Comparisons of waveforms extracted from Teukolsky wave spacetimes with the two choices of tetrad match very closely when this rescaling is taken into account.

${ }^{6}$ In addition, because, for example, the inner product $\oint_{-2} \bar{Y}_{22}(\theta, \phi)_{0} Y_{l 2}(\theta, \phi) d \Omega \neq 0$ for all $l \geq 2$, it is impossible to construct the spin-weight -2 components from spin-weight 0 components in problems lacking axial symmetry, unless one computes spherical harmonic components at all values of $l$ in the spin-weight 0 basis.
}

\section{B. Numerical Results}

For the head-on collision simulations, we use a box-inbox mesh refinement scheme similar to that employed in Section IIIB We place our outer boundary at coordinate distance $128 M$, and place refinement boundaries at $4 M$, $8 M, 16 M, 32 M$, and $64 M$ (six levels total). We find that in the non-zero shift case, we need to modify our matching condition at the refinement boundaries; this will be the topic of a forthcoming paper. We evolve only one octant of our spacetime, using appropriate symmetry boundary conditions to mimic a full grid. Because of the octant symmetry conditions used, only one of the black holes appears in our evolved numerical grid. (The other is accounted for by the symmetry boundary conditions.) We ran simulations at three resolutions with innermost resolutions of $M / 16, M / 24$, and $M / 32$ in order to perform convergence tests.

We excise a cubical region centered on each puncture. The cube has sides of length $0.23125 M$. The size, shape, and location of the excision region remains fixed during the course of the run.

In these simulations, we extract our waveforms at $r=$ $20 M, 30 M, 40 M$, and $50 M$. Since this is a non-linear problem, we are forced to use spheres at larger radii than in the Teukolsky wave simulations in order to ensure that the signals are being extracted in the wave zone.

Using the data from these three resolutions, we were able to evaluate the convergence behavior of our waveform. Fig. 4 shows a three-point convergence plot for the $l=2, m=0$ (spin-weight -2 ) component of $\Psi_{4}$ extracted from our simulations. In the three point convergence graphs, we show $\left(\Psi_{4}^{(\text {Med })}-\Psi_{4}^{(\text {High })}\right)$ and $7\left(\Psi_{4}^{(\text {Low })}-\Psi_{4}^{(\text {Med })}\right) / 20$, which should coincide, up to the effect of higher order error terms, for our second order accurate code, and our choice of relative grid spacings. ${ }^{7}$ The agreement is impressive. Panel A shows the convergence of the $l=2, m=0$ component of $\Psi_{4}$ as a function of time and as extracted at radius $r=20 M$. Panel B shows the same comparison for the extraction radius at $r=40 M$. We observe that there is a slight degradation of the signal at larger radii, but that this is to be expected because the larger radii are located in coarser regions of our grid.

In Fig. [5] we show the $l=2, m=0$ components of $\Psi_{4}$ as extracted at all four radii. As with the Teukolsky wave, we show, in Panel $\mathrm{A}$, the raw waveforms plotted as a function of time, and, in Panel B, the scaled and shifted waveforms. Again note the excellent agreement

\footnotetext{
7 The unusual convergence factor

$$
\frac{7}{20}=\frac{(1 / 3)^{2}-(1 / 4)^{2}}{(1 / 2)^{2}-(1 / 3)^{2}}
$$

is consistent with our simulations, which have resolutions in the
} ratio $1 / 2: 1 / 3: 1 / 4$. 


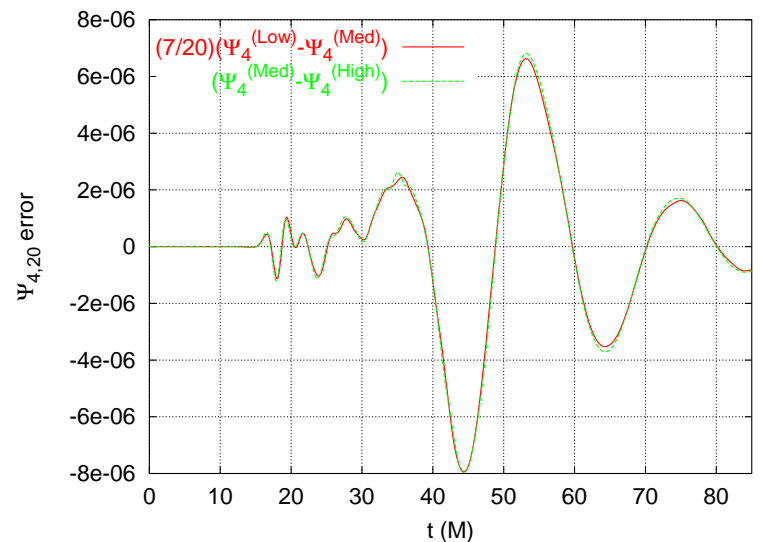

(A)

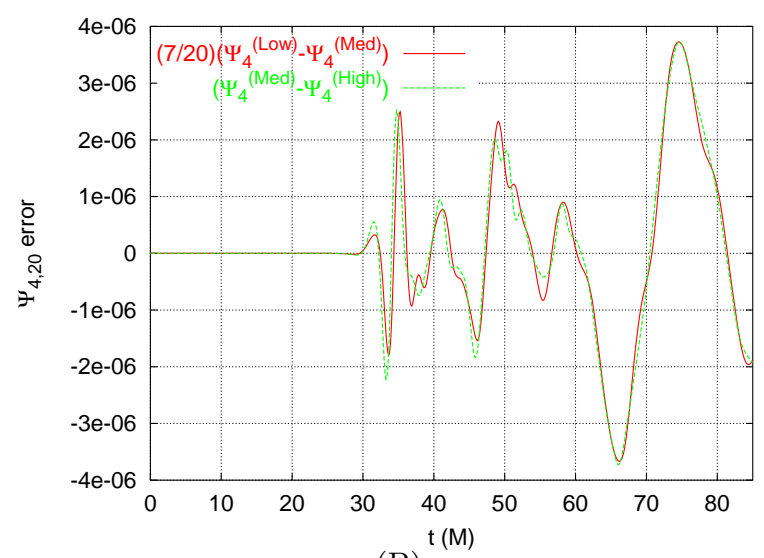

(B)

FIG. 4: A convergence plot of the $l=2, m=0$ (spin-weight -2) component of $\Psi_{4}$ for the equal mass black hole head-on collision problem. Panel A shows the convergence at the $r=$ $20 M$ extraction radius, and Panel $\mathrm{B}$ shows the convergence at the $r=40 M$ extraction radius. Note that, consistent with the coarser resolution at larger radii, there is a slight decrease in the agreement between the scaled errors in Panel B, but that the agreement is still excellent.

between waveforms extracted at different radii manifest in Panel B.

It is important to note that our scaling and shifting procedure is more complicated in the head-on collision case than it was in the Teukolsky wave case. In the head-on case, we need to account for the fact that that the coordinate speed of light is not spatially constant due to the presence of singularities. We estimate the appropriate scaling factor and time shift by (1) assuming that we extract our waves far enough from the binary system that the metric is approximately Schwarzschild, (2) using the corresponding Schwarzschild radius

$$
R=r\left(1+\frac{M}{2 r}\right)^{2}
$$

as the scale factor rather that the simulation's coordinate

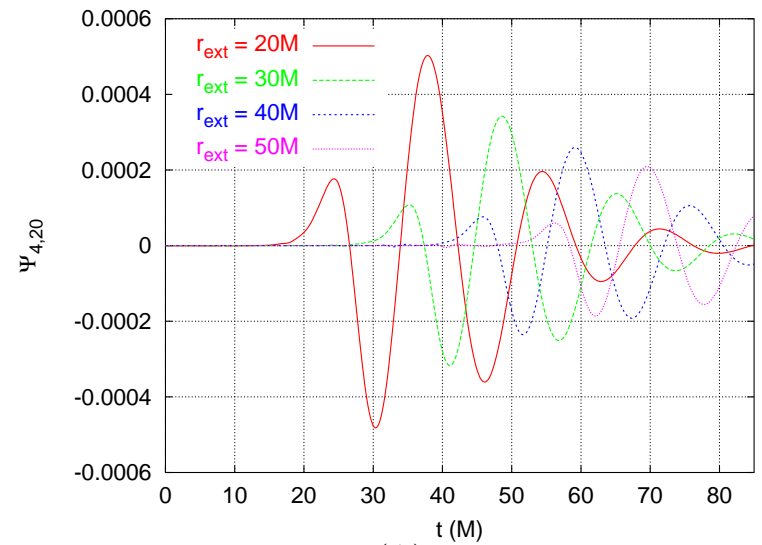

(A)

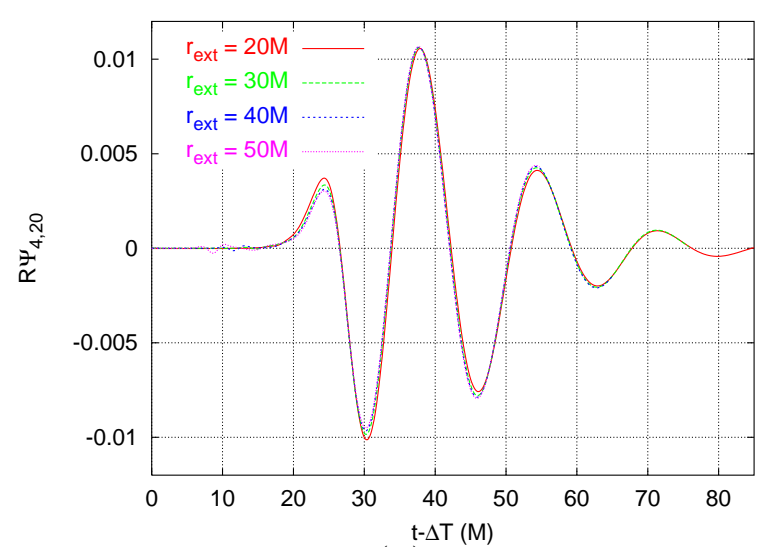

(B)

FIG. 5: A comparison between the $l=2, m=0$ components of $\Psi_{4}$ as computed at distinct radii. The waveforms have been scaled-up by $r$ and shifted to the innermost extraction radius, $r=20 M$. In order to account for the fact that our lapse is not geodesic, we scale the waveforms by an approximate Schwarzschild radius $R=r(1+M /(2 r))^{2}$ rather than the simulation's radial coordinate $r$, and shift in time by the lighttravel time in a Schwarzschild background.

radius $r$, and (3) computing the time shift

$$
\Delta T=\left[R^{\prime}+2 M \ln \left(R^{\prime} / 2 M-1\right)\right]_{R^{\prime}=R_{0}}^{R}
$$

in terms of our reference (Schwarzschild) radius $R_{0}$. The expression in square brackets is often called the "tortoise coordinate." In the case of Fig. 5. $R_{0}=R(20 M)=$ $21.0125 \mathrm{M}$.

Eq. (13) was derived in Ref. 20] (among other places), and is simply the coordinate conversion between the isotropic radial coordinate $r$ and the Schwarzschild radius $R$ for a single, spinless black hole. It is, strictly speaking, only applicable to our simulation on the initial time slice. At later times, our slices differ from the Schwarzschild slices due to our choice of lapse $\alpha$; far enough from the black holes, the slicings should be consistent and Eq. (13) should be a better and better approximation.

Eq. (14) gives the Schwarzschild coordinate time $\Delta T$ 
required for an outgoing, radial, null ray to travel from radial coordinate $R_{0}$ to radial coordinate $R$. It is also a good approximation to our coordinate time difference $\Delta t$ in the limit of large radius.

Noting that both Eq. (13) and Eq. (14) are large-radius approximations to the appropriate scale factor and time shift, it is interesting to see that, although the waveforms extracted at all radii shown in Fig. 5 agree very well, the extractions at the two farthest radii, $r=40 M$ and $r=50 M$, are extremely consistent. This is especially apparent in the first peak at $t-\Delta T \approx 25 M$ and first trough at $t-\Delta T \approx 30 M$.

In addition to checking the convergence of our waveforms and verifying consistency between the waveforms computed at different radii as shown above, we also verified that the four "sanity" checks enumerated at the end of Section IVA are satisfied, namely that both the real and imaginary parts of $\Psi_{4}$ have all applicable symmetries, and that the imaginary part converges to zero with increasing resolution. The combination of all of these checks gives us confidence in our results.

Since we do not have an analytic solution for this problem, we are not able to directly compute the error in our simulation. As a first estimate of the accuracy that we achieve, we compute the ratio between the peak error, as computed by the difference between the high and medium resolution waveforms, and the peak amplitude of the high resolution waveform. In the worst case, our extraction at $r=50 M$, this ratio is approximately $2 \%$.

\section{DISCUSSION}

We have presented a complete approach to computing gravitational radiation via the Newman-Penrose formalism given a numerically evolved solution to the $3+1$ Einstein equations. We validated our approach on the linear Teukolsky wave problem, for which we have an analytic solution. This allowed us to very carefully study the convergence behavior of our results, and to precisely measure the accuracy of the code.

Starting from the Teukolsky wave results, and combined with the technologies of mesh refinement and a spherical harmonic decomposition algorithm compatible with a non-uniform grid, we have also demonstrated that we are able to compute highly convergent waveforms generated by genuinely non-linear sources at sufficiently far distances to be considered in the wave zone. At the same time we locate the boundary of the computational domain at a sufficient distance to causally disconnect it from the extraction region and still compute several cycles of the waveform.

The high quality of our waveforms, taken together with the consistency between waveforms extracted at various radii and with different geometric relationships to our mesh refinement boundaries, also validates our particular choices for algorithms. It specifically indicates that our treatment of the mesh refinement boundary condi- tions does not introduce significant numerical errors into our simulation, and that our spherical harmonic decomposition algorithm, given good data on a non-uniform grid, does not introduce any significant errors related to the relative positions of the extraction radii and the refinement boundaries in the grid.

The work described in this paper sets the stage for us to study more interesting astrophysical cases. We are currently extending these studies to non-equal mass collisions and inspirals, in which we will extend our existing mechanisms to compute, based on the spherical harmonic components of our waveforms, the energy and momentum radiated by these systems. We also hope that these results and these well-defined test cases can serve as a basis for future code validations and comparisons much in the spirit of Ref. [12].

\section{Acknowledgments}

We would like to thank Charles Misner for providing advice as we implemented his algorithm, and Richard Matzner who provided valuable guidance in the development of these techniques. We also thank Breno Imbiriba, who has contributed to the recent development of the Hahndol code. We gratefully acknowledge the Commodity Cluster Computing Project (NASA-GSFC) and Project Columbia (NASA Advanced Supercomputing Division, NASA-Ames), which provided computing resources for the simulations described here. This work was supported in part by NASA Space Sciences grant ATP02-0043-0056. DRF and JvM were also supported in part by the Research Associateship Programs Office of the National Research Council.

\section{APPENDIX A: SUMMARY OF MISNER'S METHOD}

This appendix provides a more detailed look at the Misner algorithm for computing spherical harmonic components of a function represented on a cubic grid. Additional details can be found in Misner's original paper, Ref. 15. A detailed discussion of the truncation error as a function of the algorithm's parameters can be found in Refs. 16, 17.

Recall that the problem is to compute the spherical harmonic components $\Phi_{l m}$, defined by Eq. (4), of a function $\Phi$ that is known only on vertices of a cubic lattice. (In this appendix, we suppress the spin-weight index $s$.) In order to begin, two definitions are need. First define a family of radial functions

$$
R_{n}(r ; R, \Delta)=r^{-1} \sqrt{\frac{2 n+1}{2 \Delta}} P_{n}\left(\frac{r-R}{\Delta}\right)
$$

in terms of the usual Legendre polynomials $P_{n}$. Here $R$ and $\Delta$ are parameters that will be associated with the 
radius at which the spherical harmonic decomposition is desired and half of the thickness of a shell centered on that radius. From this, define

$$
Y_{n l m}(r, \theta, \phi)=R_{n}(r) Y_{l m}(\theta, \phi)
$$

which form a complete, orthonormal set with respect to the inner product

$$
\langle f \mid g\rangle=\int_{S} \bar{f}(x) g(x) d^{3} x
$$

on the shell $S=\{(r, \theta, \phi) \mid r \in[R-\Delta, R+\Delta]\}$. Note also that, because the functions $R_{n}$ form a complete set in the radial direction,

$$
\Phi_{l m}(t, R)=\int \rho(r ; R, \Delta) \bar{Y}_{l m}(\theta, \phi) \Phi(r, \theta, \phi) d^{3} x
$$

with

$$
\rho(r ; R, \Delta)=\sum_{n=0}^{\infty} R_{n}(R ; R, \Delta) R_{n}(r ; R, \Delta),
$$

and that

$$
\rho(r ; R, \Delta)=r^{-2} \delta(R-r)
$$

is a delta function. (Compare Eq. (A4) to Eq. (4).)

On a finite grid $\Gamma$, the inner product Eq. (A3) will have the form

$$
\langle f \mid g\rangle=\sum_{x \in \Gamma} \bar{f}(x) g(x) w_{x}
$$

where each point has some weight $w_{x}$. This weight was given the form

$$
w_{x}= \begin{cases}0 & |r-R|>\Delta+h / 2 \\ h^{3} & |r-R|<\Delta-h / 2 \\ (\Delta+h / 2-|R-r|) h^{2} & \text { otherwise }\end{cases}
$$

by Misner, where $h$ is the grid spacing. Only cases with $\Delta>h / 2$ are considered. This means, roughly, that points entirely within the shell $S$ are weighted by their finite volume on the numerical grid, points entirely outside of the shell $S$ have zero weight, and points near the boundary are weighted according to the fraction of their volume inside $S$.

With the numerical inner product Eq. (A7), and letting capital Roman letters $A=(n l m)$ represent index groups, the $Y_{A}$ are no longer orthonormal. Their inner product

$$
\left\langle Y_{A} \mid Y_{B}\right\rangle=G_{A B}=\bar{G}_{B A}
$$

forms a metric for functions on the shell. (Although a priori this matrix appears to be complex valued, it is actually real-symmetric and sparse, cf. Refs. 16, 17]. For now it suffices to follow Misner in denoting it as generically Hermitian.) The inverse to this metric $G^{A B}$ can be used to raise indices on functions defined on the sphere.
Making use of this new metric, and with some further analysis, the approximation for the spherical harmonic coefficients

$$
\Phi_{l m}(t, R)=\sum_{x \in \Gamma} \bar{R}_{l m}(x ; R) w_{x} \Phi(t, x)
$$

follows with

$$
R_{l m}(r ; R)=\sum_{n=0}^{N} \bar{R}_{n}(R) Y^{n l m}(r, \theta, \phi)
$$

in terms of $Y^{A}=G^{B A} Y_{B}$, not $Y_{A}$.

Note that one need only store the combination $w_{x} \bar{R}_{l m}$ at points where $w_{x} \neq 0$ in order to compute the spherical harmonic components. This buries all the details of the computation in a relatively small table of numbers, and, since these numbers are not time dependent, this calculation need be done only once per simulation.

\section{APPENDIX B: TEUKOLSKY WAVE SOLUTION}

The general form of the spacetime metric for the Teukolsky wave solution [23] is given by Eq. (5). The angular function are

$$
\begin{aligned}
f_{r r} & =2-3 \sin ^{2} \theta \\
f_{r \theta} & =-3 \sin \theta \cos \theta \\
f_{r \phi} & =0 \\
f_{\theta \theta}^{(1)} & =3 \sin ^{2} \theta \\
f_{\theta \theta}^{(2)} & =-1 \\
f_{\theta \phi} & =0 \\
f_{\phi \phi}^{(1)} & =-f_{\theta \theta}^{(1)} \\
f_{\phi \phi}^{(2)} & =3 \sin ^{2} \theta-1
\end{aligned}
$$

for the $l=2, m=0$ case. The remaining functions

$$
\begin{array}{ll}
A=3\left(\frac{F^{(2)}}{r^{3}}+\frac{3 F^{(1)}}{r^{4}}+\frac{3 F}{r^{5}}\right) & \quad(\mathrm{B} 2 \mathrm{a}) \\
B=-\left(\frac{F^{(3)}}{r^{2}}+\frac{3 F^{(2)}}{r^{3}}+\frac{6 F^{(1)}}{r^{4}}+\frac{6 F}{r^{5}}\right) & (\mathrm{B} 2 \mathrm{~b}) \\
C=\frac{1}{4}\left(\frac{F^{(4)}}{r}+\frac{2 F^{(3)}}{r^{2}}+\frac{9 F^{(2)}}{r^{3}}+\frac{21 F^{(1)}}{r^{4}}+\frac{21 F}{r^{5}}(\mathrm{~B}) 2 \mathrm{c}\right)
\end{array}
$$

are written in terms of a free generating function $F=$ $F(t-r)$, which we choose to have the form given by Eq. (6). The notation

$$
F^{(n)}=\left[\frac{d^{n} F(x)}{d x^{n}}\right]_{x=t-r}
$$

denotes various derivatives. Taking $F$ as a function of $t-$ $r$ corresponds to outgoing waves. To generate an ingoing solution, change the argument of $F$ to $t+r$, and change, 
in Eq. (B2), the sign in front of all of the terms with odd numbers of derivatives. (Note that the description of how ingoing waves are constructed in Ref. 24] contains an error. The description here, which matches the original reference, Ref. [23], is correct.)

The Weyl scalar $\Psi_{4}$ for this spacetime is computed from the definition Eq. (2) using the Kinnersley tetrad, Eq. (7), and noting that, of the twelve non-zero components of the Riemann tensor associated with the metric Eq. (5), only

$$
\begin{aligned}
R_{t \theta t \theta}= & -\frac{3}{2} r^{2} \sin ^{2} \theta \frac{\partial^{2} C}{\partial t^{2}}+\frac{1}{2} r^{2} \frac{\partial^{2} A}{\partial t^{2}} \\
R_{t \phi t \phi}= & \frac{3}{2} r^{2} \sin ^{4} \theta\left(\frac{\partial^{2} C}{\partial t^{2}}-\frac{\partial^{2} A}{\partial t^{2}}\right) \\
& +\frac{1}{2} r^{2} \sin ^{2} \theta \frac{\partial^{2} A}{\partial t^{2}} \\
R_{t \theta r \theta}= & -\frac{1}{8} r^{3} \sin ^{2} \theta\left(3 \frac{\partial^{3} B}{\partial t^{3}}+\frac{\partial^{3} A}{\partial t^{3}}\right) \\
R_{r \phi r \phi}= & -\sin ^{2} \theta R_{t \theta t \theta} \\
R_{r \theta r \theta}= & -\frac{1}{\sin ^{2} \theta} R_{t \phi t \phi} \\
R_{t \phi r \phi}= & -\sin ^{2} \theta R_{t \theta r \theta}
\end{aligned}
$$

[1] B. Barish, in 31], p. 3.

[2] P. Fritschel, in 31], p. 15.

[3] B. F. Schutz, in The Astrophysics of Gravitational Wave Sources, edited by J. M. Centrella (American Institute of Physics, Melville, New York, 2003), pp. 3-26.

[4] P. Bender, A. Briller, I. Ciufolini, A. M. Cruise, C. Cutler, K. Danzmann, F. Fidecaro, W. M. Folkner, J. Hough, P. McNamara, et al. (1998), on-line resource [cited July 11, 2004] http://www.srl.caltech.edu/lisa/documents

[5] K. Camarda and E. Seidel, Phys. Rev. D 57, 3204 (1998).

[6] J. Baker, S. Brandt, M. Campanelli, C. O. Lousto, E. Seidel, and R. Takahashi, Phys. Rev. D 62, 127701 (2000).

[7] M. Alcubierre, W. Benger, B. Brügmann, G. Landerman, L. Nerger, E. Seidel, and R. Takahashi, Phys. Rev. Lett. 87, 271103 (2001).

[8] M. Alcubierre, B. Brügmann, D. Pollney, and E. S. adn Ryoji Takahashi, Phys. Rev. D 64, 061501 (2001).

[9] M. Alcubierre, B. Brügmann, P. Diener, M. Koppitz, D. Pollney, E. Seidel, and R. Takahashi, Phys. Rev. D 67, 084023 (2003).

[10] M. Alcubierre, B. Brügmann, P. Diener, F. Herrmann, D. Pollney, E. Seidel, and R. Takahashi (2004), grqc/0411137.

[11] U. Sperhake, B. Kelly, P. Laguna, K. L. Smith, and E. Schnetter (2005), gr-qc/0503071.

[12] M. Alcubierre, G. Allen, C. Bona, D. Fiske, T. Goodale, F. S. Guzmán, I. Hawke, S. H. Hawley, S. Husa, M. Koppitz, et al., Class. Quant. Grav. 21, 589 (2004).

[13] S. Chandrasekhar, The Mathematical Theory of Black Holes (Oxford University Press, 1992).

[14] J. Baker, M. Campanelli, and C. O. Lousto, Phys. Rev. D 65, 044001 (2002). contribute to the sum.

[15] C. W. Misner, Class. Quant. Grav. 21, S243 (2004).

[16] D. R. Fiske, Ph.D. thesis, University of Maryland, College Park (2004), http://hdl.handle.net/1903/1805

[17] D. R. Fiske (2004), gr-qc/0412047.

[18] M. Shibata and T. Nakamura, Phys. Rev. D 52, 5428 (1995).

[19] T. W. Baumgarte and S. L. Shapiro, Phys. Rev. D 59, 024007 (1999), gr-qc/9810065.

[20] B. Imbiriba, J. Baker, D.-I. Choi, J. Centrella, D. R. Fiske, J. D. Brown, J. van Meter, and K. Olson, Phys. Rev. D 70, 124025 (2004), gr-qc/0403048.

[21] S. A. Teukolsky, Phys. Rev. D 61, 087501 (2000), grqc/9909026.

[22] P. MacNeice, K. Olson, C. Mobarry, R. de Fainchtein, and C. Packer, Comput. Phys. Comm. 126, 330 (2000).

[23] S. A. Teukolsky, Phys. Rev. D 26, 745 (1982).

[24] D.-I. Choi, J. D. Brown, B. Imbiriba, J. Centrella, and P. MacNeice, J. Comp. Phys. 193, 398 (2004).

[25] W. Kinnersley, J. Math. Phys. 10, 1195 (1969).

[26] J. Baker, B. Brügmann, M. Campanelli, and C. O. Lousto, Class. Quant. Grav. p. L149 (2000).

[27] M. C. J. Baker, C. O. Lousto, and R. Takahashi, Phys. Rev. D 65, 124012 (2002).

[28] B. Brügmann, Phys. Rev. D 54, 7361 (1996).

[29] D. Brill and R. Lindquist, Phys. Rev. 131, 471 (1963).

[30] D. Shoemaker, K. Smith, U. Sperhake, P. Laguna, E. Schnetter, and D. Fiske, Class. Quant. Grav. 20, 3729 (2003), gr-qc/0301111.

[31] J. M. Centrella, ed., Astrophysical Sources for GroundBased Gravitational Wave Detectors (American Institute of Physics, Melville, New York, 2001). 